\title{
MULTIPLE-VALUED STRUCTURES OF INTELLECTUAL SYSTEMS
}

\section{Mikhail Bondarenko, Irina Vechirskaya, Grigoriy Chetverikov}

\begin{abstract}
The basic construction concepts of many-valued intellectual systems, which are adequate to primal problems of person activity and using hybrid tools with many-valued intellectual systems being two-place, but simulating neuron processes of space toting which are different on a level of actions, inertial and threshold of properties of neuron diaphragms, and also frequency modification of the following transmitted messages are created. All enumerated properties and functions in point of fact are essential not only are discrete on time, but also many-valued.
\end{abstract}

Keywords: many-valued intellectual system, level of actions, parallelism, analyze-coordination processor, processor-supervisor, knowledge base, artificial intelligence hybrid logic, multiple-valued logic, multi-state element.

ACM Classification Keywords: I.2.4. Knowledge Represetation Formalisms and Methods.

Conference: The paper is selected from XIVth International Conference "Knowledge-Dialogue-Solution" KDS 2008, Varna, Bulgaria, June-July 2008

\section{Introduction}

Construction methods of switching circuits (multiple-valued invertible heterogeneous logic elements) of the first and second kind for language systems of artificial intelligence with invertible properties concerning multiplevalued heterogeneous codes have been modified and they have received a subsequent development effort. Research of hardware-controlled means of obtained models implementation of the language and synthesis methods of multiple-valued invertible heterogeneous logic elements allowed rational ways and their relations to be determined as well as to formulate selection criteria of multiple-valued invertible heterogeneous logic elements for implementation of relations which make up the action base of language systems.

The basic construction concepts of many-valued intellectual systems (MIS), which are adequate to primal problems of person activity and using hybrid tools with many-valued coding are considered. With materialism of a point of view these concepts are agreed with the dialectic laws opened by a man and their manifestations in problems connected with creation of identification systems prediction and recognition of imagery in which the interactive operational mode is a main part of the whole complex of intellectual properties [1,2].

Those are, for example, the law of unity and struggle of contrasts - as availability in parallel operating in space and time of mechanisms both discrete, and continuous mapping objects of plants; the law of transition from quantitative changes to qualitative-quantitative changes of gradation levels of brightness and the color result in qualitative changes in mapping of objects; the law of negation of negation - as a changes and alternation of coding indications of messages about objects in neurons of a brain - from space to temporal and from two-place to many-valued.

In particular, in works the accent on the concept of neuro-physiologic and neuro-cybernetic aspects of alive brain mechanisms is made. It is connected with the following natural neuron structures from nervous cells - neurons, essentially are highly effective recognizing systems and, for this reason, is of interest not only for doctors physiologists, but also for the experts designing artificial intelligence systems. However direct transfer of research results of neuro-physiologists in engineering practice is now impossible because of a lack of an appropriate bioelectronic technology and an element basis, that has led to development and creation of a set of varieties of artificial neurons realized on the elements of the impulse technology [3, 4].

But also here there were complications because of non-adequate neuron models to a set of the demands made of MIS. Creation of neuro-like models on the basis of multiprocessor in inputting systems technology with programmed architecture, in particular, on the basis of digital integrating structures is offered as the alternative in works. Thus, retaining Neumann structure a MIS are created, being essentially two-place, but simulating neuron processes of space toting different on a level of actions, inertial and threshold properties of neuron diaphragms, 
as well as variation of recurrence frequency of transmitted messages. Though it is obvious that all enumerated properties and functions in point of fact, are, essential, not only discrete on time, but also many-valued (are discrete on a level).

\section{Structurally Functional Cell Model of a Many-Valued Intellectual System}

As the corollary, non-adequacy of used principles of coding and element basis to simulated processes entails a redundancy, complication and non evidence of used mathematical and engineering means of transformations [5], loss of a micro level of parallelism in handling expected fast acting and flexibility of restructuring without essential modifications of architecture and connections.

The originating complications [1], in creation of a many-valued intellectual system promote moving out of the adequacy concept of many-valued logic and structures of MIS creation problems with desirable properties and possibilities.

Therefore, for disclosure of use paths of a knowledge backlog in the field of many-valued coding and structures in MIS creation the conceptual structurally functional model of a MIS cell (Fig.1) is offered.

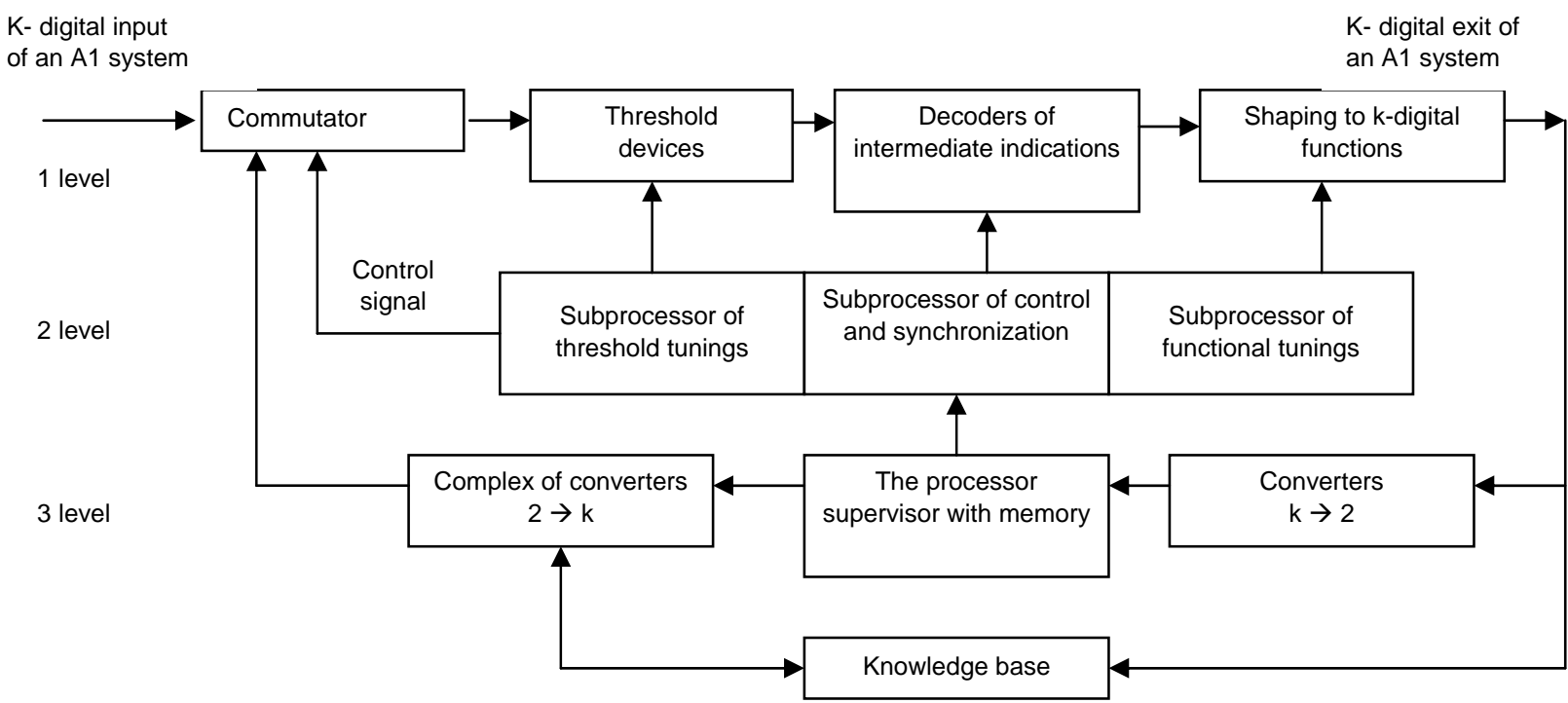

Fig. 1. A conceptual structurally functional model of a MIS cell

Each MIS is characterized by a set of functions fulfilled by blocks, which realize functions and information interchanges. In accordance with solved problems, the structurally functional cell breaks up to three hierarchical levels: functional (analytic-synthetic) - level 1; tactical (analyses-coordination) - level 2; strategic (coordination) level 3.

The MIS cell increases on a function level both on inputs, and on outputs, and it is integrated with other meshes on inputs of decoders of intermediate indications; at a tactical level - through the analyze-coordination processor; at a strategic level - through the processor-supervisor and knowledge base. The conceptual model of a MIS cell is based on the concept of symbiosis of two- and many-valued tools of data processing, therefore at a strategic level it contain complexes of converters of the data representation form - converters from a two-place code to many-valued $2 \rightarrow \mathrm{k}$ and back $2 \leftarrow \mathrm{k}$. Obviously, that their use in MIS determines, at what level the problems, are solved in what logic and with what speed (what channel capacity of MIS). Besides the application of these tools excludes necessity of an operator work with two-place translators in input - output of data.

The new principle of the computers construction is offered, in which the principle of organization of brainwork simultaneously with a principle of programmed control assumes as a basis. The principle of organization of brainwork assumes as a basis of operation of such computers, in classical element basis it will be for more to Hilbert machines than for nowadays existing Neumann machines, the basis of which is the principle of programmed control realized rather slowly. 


\section{Formalization of Construction Principles of Many-Valued Spatial Structures}

In the generalized from the two-input universal k-valued structure of a spatial type contains two recognition elements (RE), the control unit (CU), the matrix selector (MS), commutator $(C)$, and keys (K) or the digital-toanalog converter (DAC) (Fig.2).

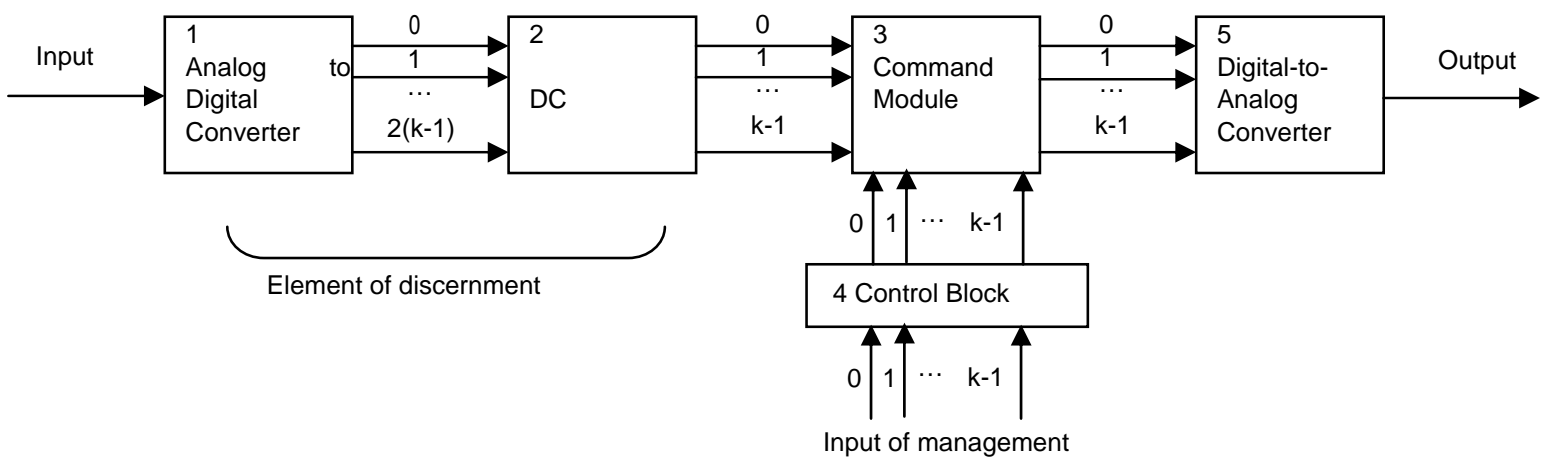

Fig. 2. Universal Multiple-Valued Functional Converter

The logic of the decoders operation in recognition elements 1,2 is described by the fallowing equation system:

$$
\begin{aligned}
& f_{0}=\left(x_{0}, x_{1}, \ldots, x_{k-1}\right)=y^{0}, \\
& f_{1}=\left(x_{0}, x_{1}, \ldots, x_{k-1}\right)=y^{1}, \\
& \ldots \ldots \ldots \ldots \ldots \ldots \ldots \ldots \ldots \ldots \ldots \ldots \ldots \ldots \ldots \ldots \ldots \ldots \ldots \ldots \ldots \\
& f_{k-1}=\left(x_{0}, x_{1}, \ldots, x_{k-1}\right)=y^{k-1} .
\end{aligned}
$$

Or in the explicit form at the algebra language of finite predicates [1]:

$$
\begin{aligned}
& y_{1,2}^{0}=\overline{x_{1}}, \\
& y_{1,2}^{1}=x_{1} \cup \overline{x_{2}}, \\
& y_{1,2}^{2}=x_{2} \cup \overline{x_{3}}, \\
& \cdots \cdots \cdots \cdots \cdots \cdots \\
& y_{1,2}^{k-1}=x_{k-1} .
\end{aligned}
$$

where $x_{i}$ and $\overline{x_{i}}(i=\overline{0, k-1})$ - signals of direct and inversion outputs of the ADC units in recognition elements 1,2 . The logic of the matrix selector is described by the following equation system:

$$
\begin{aligned}
& b_{00}=y_{1}^{0} \cup y_{2}^{0}, b_{01}=y_{1}^{0} \cup y_{2}^{1}, \ldots, b_{0(k-1)}=y_{1}^{0} \cup y_{2}^{k-1}, \\
& b_{10}=y_{1}^{1} \cup y_{2}^{0}, b_{11}=y_{1}^{1} \cup y_{2}^{1}, \ldots, b_{1(k-1)}=y_{1}^{1} \cup y_{2}^{k-1},
\end{aligned}
$$$$
b_{(k-1) 0}=y_{1}^{k-1} \cup y_{2}^{0}, b_{(k-1) 1}=y_{1}^{k-1} \cup y_{2}^{1}, \ldots, b_{(k-1)(k-1)}=y_{1}^{k-1} \cup y_{2}^{k-1},
$$

where $b_{i j}(i, j=\overline{0, k-1})$ - output logical signals of the matrix selector 4 . The commutator has two groups by $k$ inputs: the signals from the selector are applied to the first group and control signal values are applied to the second group. In the explicit from the commutator operation is described by the following system: 


$$
\begin{aligned}
& b^{k_{0}} l^{0} \cup b^{k_{0}} l^{1} \cup \ldots \cup b^{k_{0}} l^{k-1}=z^{k_{0}}, \\
& b^{k_{1}} l^{0} \cup b^{k_{1}} l^{1} \cup \ldots \cup b^{k_{1}} l^{k-1}=z^{k_{1}}, \\
& \ldots \ldots \ldots \ldots \ldots \ldots \ldots \ldots \ldots \ldots \ldots \ldots \ldots \ldots \ldots \ldots \ldots \ldots \ldots \ldots \ldots \ldots \ldots \ldots \ldots \ldots \ldots \ldots \ldots \ldots \ldots \ldots \ldots \ldots \\
& b^{k_{k-1}} l^{k-1}=z^{k_{k-1}} .
\end{aligned}
$$

As all $k$ of keys of the output shaper are constantly connected to corresponding $k$-values of output signals the function values selected by the commutator and the control unit, respectively, will arrive in the converter output (structure) in the course of variations of $k$-valued functions on the converter inputs. The process control of the logic recommutations is carried out under the action of external control signals.

\section{Composition and Decomposition of Spaces}

During the last years the problem of understanding people by the computers has become very popular. It arises with some attempts to make work conditions better. The scientists try to formalize natural language with ManyValued Intellectual Structures. The mechanism of natural language is formally described by facilities of logic mathematics. At the same time in the terms of linguistic algebra the thought is a predicate, the sentence is a formula of predicate operations algebra. Sentence semantics is described by the language of predicate algebra. Grammatical structure of the sentence is described by predicate operations algebra.

We have an algebro-logical language. Using it we can express an algebro-logical structure of natural language. Main task of logic mathematics in the science of language is to know what variant of predicate operations algebra was realized in the natural language. Therefore logic mathematics should strengthen its algebraic tools.

Let's consider the space $\mathrm{S}$

$$
S\left(x_{1}, x_{2}, \ldots, x_{n}, y\right)=1 \text {, }
$$

where $\mathrm{y}$ is a point of the space, $\left(x_{1}, x_{2}, \ldots, x_{n}, y\right)$ is its coordinate representation.

Suppose $S\left(x_{1}, x_{2}, \ldots, x_{n}, y\right)$ as a predicate, which is determined on the cartesian product $A \times B$. Let's name the set $A=A_{1} \times A_{2} \times \ldots \times A_{n}$ as a coordinate system of space, $\mathrm{B}$ is a space support.

Theorem. Every predicate of disjunktively - conjunctive algebra can be represented as

$$
P\left(x_{1}, x_{2}, \ldots, x_{m}\right)=\underset{a_{1}, a_{2}, \ldots, a_{m} \in U}{\vee} P\left(a_{1}, a_{2}, \ldots, a_{m}\right) x_{1}^{a_{1}} x_{2}^{a_{2} \ldots x_{m}^{a_{m}} .}
$$

Let's consider a predicate $S\left(x_{1}, x_{2}, \ldots, x_{n}, y\right)$ :

$$
\begin{aligned}
& x_{1}: S\left(x_{1}, x_{2}, \ldots, x_{n}, y\right)=\underset{a \in A_{1}}{\vee} x_{1}^{a} S\left(a, x_{2}, \ldots, x_{n}, y\right), \\
& S_{a}\left(x_{2}, \ldots, x_{n}, y\right)=S\left(a, x_{2}, \ldots, x_{n}, y\right) .
\end{aligned}
$$

Let's illustrate space exfoliation.

An example. Let's consider the short form of adjective

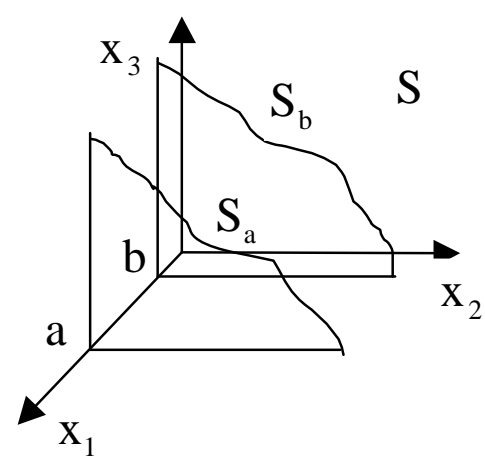

Fig. 3. Graphical interpretation of morphological predicate 


$$
\begin{aligned}
& B^{\prime}=\{\text { мил, мила, мило, миль }\}, \\
& A_{1}=\{e, \mu\}, A_{2}=\{y, \sigma\}, A_{3}=\{\varkappa, \mu, c\}, B=\{, a, o, b l\} .
\end{aligned}
$$

Morphological predicate is represented in the following form:

$$
\begin{aligned}
& S\left(x_{1}, x_{2}, x_{3}, y\right)=x_{1}^{e} x_{2}^{y} x_{3}^{M} y-\vee x_{1}^{e} x_{2}^{\sigma} x_{3}^{\varkappa} y^{a} \vee x_{1}^{e} x_{2}^{y} x_{3}^{c} y^{o} \vee x_{1}^{M} x_{2}^{\sigma} y^{b l} ; \\
& \text { 1) } x_{1}: S_{e}\left(x_{2}, x_{3}, y\right)=x_{2}^{y} x_{3}^{M} y-\vee x_{2}^{\sigma} x_{3}^{\varkappa c} y^{a} \vee x_{2}^{y} x_{3}^{c} y^{o}, S_{\mathcal{M}}\left(x_{2}, x_{3}, y\right)=x_{2}^{\sigma} y^{b l} ; \\
& \text { 2) } x_{2}: S_{y}\left(x_{1}, x_{3}, y\right)=x_{1}^{e} x_{3}^{\mathcal{M}} y^{-} \vee x_{1}^{e} x_{3}^{c} y^{o}, S_{\sigma}\left(x_{1}, x_{3}, y\right)=x_{1}^{e} x_{3}^{\varkappa c} y^{a} \vee x_{1}^{\mathcal{M}} y^{b l} ; \\
& \text { 3) } x_{3}: S_{\mathcal{H}}\left(x_{1}, x_{2}, y\right)=x_{1}^{e} x_{2}^{\sigma} y^{a} \vee x_{1}^{M} x_{2}^{\sigma} y^{b l}, S_{\mathcal{M}}\left(x_{1}, x_{2}, y\right)=x_{1}^{e} x_{2}^{y} y-\vee x_{1}^{M} x_{2}^{\sigma} y^{b l}, \\
& S_{c}\left(x_{1}, x_{2}, y\right)=x_{1}^{e} x_{2}^{y} y^{o} \vee x_{1}^{M} x_{2}^{\sigma} y^{b l} .
\end{aligned}
$$

We have 11 predicates of letters recognition in 1), 2) and 18 - in 3). This decomposition shows that the "род" in a certain sense is older than "ударность" and "число".

Composition is shown in the following form (we used idempotent and true laws):

$$
\begin{aligned}
& S\left(x_{1}, x_{2}, x_{3}, y\right)=x_{1}^{e}\left(x_{2}^{y} x_{3}^{M} y-\vee x_{2}^{\sigma} x_{3}^{* c} y^{a} \vee x_{2}^{y} x_{3}^{c} y^{o}\right) x_{1}^{M} x_{2}^{\sigma} y^{b l} \vee x_{2}^{y}\left(x_{1}^{e} x_{3}^{M} y-\vee x_{1}^{e} x_{3}^{c} y^{o}\right) \vee \\
& \vee x_{2}^{\sigma}\left(x_{1}^{e} x_{3}^{\varkappa c} y^{a} \vee x_{1}^{M} y^{b l}\right) \vee x_{3}^{\varkappa c}\left(x_{1}^{e} x_{2}^{\sigma} y^{a} \vee x_{1}^{M} x_{2}^{\sigma} y^{b l}\right) \vee x_{3}^{M}\left(x_{1}^{e} x_{2}^{y} y^{-} \vee x_{1}^{M} x_{2}^{\sigma} y^{b l}\right) \vee \\
& \vee x_{3}^{c}\left(x_{1}^{e} x_{2}^{y} y^{o} \vee x_{1}^{M} x_{2}^{\sigma} y^{b l}\right)=x_{1}^{e} x_{2}^{y} x_{3}^{M} y-\vee x_{1}^{e} x_{2}^{\sigma} x_{3}^{\varkappa c} y^{a} \vee x_{1}^{e} x_{2}^{y} x_{3}^{c} y^{o} \vee x_{1}^{M} x_{2}^{\sigma} y^{b l} \text {. }
\end{aligned}
$$

Therefore we can analyse the mechanism of language evolution.

Composition and decomposition of space can be used in language formalization.

Thus, main principles of representation of multidimensional relations as a composition of binary relations were discussed. The formal description of adjective's endings was generated. It was made an attempt to build a relational model of process of ending's formation as a construction of binary tables.

\section{Conclusion}

On the basis of general principles and methods of universality hybridism and parallelism (speed) of k-valued spatial structures a new class of universal functional converters - switching circuits of the third kind which allows to implement analysis, normalization and synthesis of Ukrainian language morphology problems has been created.

The modification of the conventional tables of truth for multiple-valued invertible heterogeneous codes, connected by predicated equations allowing to represent these tables in the compact form is proposed. In so doing, a number of elements of the modified table in $3^{\prime}$ times, where variable $p$ is a depth of decomposition.

It is shown that the algebra and logic method of invertible switching, circuits synthesis of the first kind is propagated to a case of heterogeneous codes, predetermined by predicate equations [6].

The utility of the transfer to switching circuits of the second kind in a number of variables in the initial predicate equation more 8 in a digit number of variables not exceeding 6 , which is characteristic for problem solving of morphological analysis and synthesis is justified [7-8].

It is found that switching circuits of direct and back action synthesized on the basis of logic elements and modules possess a number of characteristic properties and properties of full and partial regeneration, contradiction detection in input signals.

The problem solving of principles formalization of the structure organization of computing tools, thus ensures construction of the newest concept for systems of an artificial intelligence; application of space and temporal parallelism at structural and algorithmic levels; creation of procedural an function languages, parallel machines of knowledge bases and the interface. The problem solving of organization principles formalization of universal $k$ - 
valued structures of a spatial type by tools of predicate and hybrid logic will ensure construction of a modern concept for artificial intelligence systems, application of spatial parallelism at structured and algorithmic levels; creation of functional languages of parallel machines of knowledge basis; application of symbiosis of two- and many-level heterogeneous coding.

One of circuit for realization of multiple-valued elements is the frequency-harmonic multi-state element which states are coding by amplitude and frequency. This element was made by thin film technology as hybrid integrated circuit.

This paper is devoted to building of formalization methods of the relation. It is a main tools for realization of ManyValued Intellectual System which focused on parallel information processing and its program realization.

The utilization of developed outlet's instruction strategics is minimized the search time and this is to increase the efficiency of outlet. Developed logical algebra mathematical tool is simplified of given types problem for both knowledge engineer and user.

\section{Bibliography}

[1] Bondarenko M., Chetverikov G., Karpukhin A. Analiz problemi sozdaniya novich tekhnicheskich sredstv dlya realizatsii lingvisticheskogo interfeisa. Proc of the $10^{\text {th }}$ International Conference KDS - 2003, Varna, Bulgaria, June 16-26, 2003, pp.78-92.

[2] Bondarenko M., Chetverikov G., Deyneko Zh. Application of a numerically - analytical method for simulation of non-linear resonant circuits. 10th International Conference "Mixed Design Of Integrated Circuits And Systems" (MIXDES 2003), Lodz, Poland, 26-29 June 2003, pp.131-133.

[3] Bondarenko M., Chetverikov G., Leshchinsky V. Synthesis Methods of Multiple-valued Structures of Bilogical Networks // Proc. Of the 12th International Conf. "Mixed design of integrated circuits and systems" (MIXDES 2005), Krakow (Polska), 2005. - Pp. 201-204.

[4] Bondarenko M., Chetverikov G., Karpukhin A. Structural Synthesis of Universal Multiple-Valued Structures of Artificial Intelligence Systems // Proc. Of the 9th World Multi-Conference in Systemics,Cybernetics and Informatics (WMSCI 2005). - Orlando, Florida (USA), 2005. Vol. VII. - Pp. 127-130.

[5] Bondarenko M.F., Konoplyanko Z.D., Chetverikov G.G. Theory fundamentals of multiple-valued structures and coding in artificial intelligence systems.- Kharkiv: Factor-druk, 2003. - 336 p.

[6] Bondarenko M.F., Z.D. Konopljanko, G.G. Chetverikov. Osnovy teorii synteza nadshvydkodiuchikh structur movnykh sistem shtuchnogo intellectu, Monografia. - K.: IZMN, 1997. - $386 \mathrm{~s}$.

[7] Bondarenko M.F., S.V. Lyahovets, A.V. Karpukhin, G.G. Chetverikov. Sintez shvidkodiuchikh structur lingvistichnich ob'ekhtiv., Proc. of the 9th International Conference KDS - 2001, St.Peterburg, Russia, 2001, s. 121-129.

[8] Bondarenko M.F., V.N. Bavykin, I.A. Revenchuk, G.G. Chetverikov. Modeling of universal multiple-valued structures of artificial intelligence systems, Proc. of the 6th International Workshop "MIXDES'99", Krakow, Poland, 17-19 June 1999, pp. 131-133.

\section{Authors' Information}

Mikhail Bondarenko- Professor, Doctor of Technical Sciences; Rector of the State National University of RadioElectronics P.O. Box 14, Lenin's avenue, Kharkov, 61166; Ukraine.

Irina Vechirskaya - Researcher; State National University of Radio-Electronics P.O. Box 14, Lenin's avenue, Kharkov, 61166, Ukraine; e-mail: ira se@list.ru.

Grigoriy Chetverikov - Professor, Doctor of Technical Sciences; State National University of Radio-Electronics P.O. Box 14, Lenin's avenue, Kharkov, 61166, Ukraine; e-mail: chetvergg@kture.kharkov.ua. 